\title{
PARASITIC ENERGY LOSS IN THE LEP SUPERCONDUCTING CAVITIES
}

\author{
G. Arduini, O. Brüning, G. Cavallari, P. Gayet, G. Geschonke, E. Häbel, A. Hofmann, M. Jimenez, \\ O. Meincke, G. Morpurgo, G. Roy, H. Schmickler, J. Uythoven, A. Wagner, B. Zotter, \\ CERN, 1211 Geneva 23, Switzerland
}

\begin{abstract}
The energy loss of bunches in the LEP superconducting (SC) cavities has been determined by measuring the closed orbit as a function of current with the beam position monitors located at finite dispersion. This method has already been used in earlier experiments to determine the distribution of the longitudinal impedance of different parts of LEP. In the present experiment the energy loss in two straight sections, containing only SC cavities, was compared with that in sections having both copper cavities and SC cavities. The results confirm the impedance calculations for the two types of cavities. The accuracy of the measurements was considerably improved by determining simultaneously the orbits of bunches with different currents. At the same time with these beam-based impedance measurements, the power dissipation was observed directly by local temperature monitors in different elements: the inter-cavity bellows inside the cryostat, the warm intermodule bellows, and Ferrite absorbers which were installed in two places to reduce the energy leaking out of cavities. These observations were correlated with the change of cryogenics power consumption, and showed an unexpected dependence of energy loss on beam energy.
\end{abstract}

\section{INTRODUCTION}

The total energy loss of the beam in a storage ring can be determined by measuring the change of the synchronous phase as function of bunch current. This was done in LEP by comparing the output from a fast intensity monitor with the signal from an RF cavity. In a second method, the longitudinal bunch position was measured with a streak camera triggered by the RF-voltage [1]. Since the loss factor is expected to be a strong function of bunch length, all these measurements were carried out for a number of different RF voltages which changes this length.

The spatial distribution of energy loss in LEP has been determined by measuring the change of the closed orbit as function of bunch current, using beam position monitors in dispersive regions [2]. A similar method has also recently been used for energy loss measurements at KEK [3]. These measurements permit independent determination of the impedances of RF-cavities and of distributed elements in the arcs. By measuring simultaneously orbits of bunches with different currents, rather than measuring the same bunch after changing its current, errors due to fast orbit drifts could be avoided - and the accuracy of the measurements was thereby improved considerably.

During the last few years, a large number of SC cavities - grouped in "modules" of four four-cell cavities - has been installed in LEP, in addition to the already existing five-cell copper cavities. Due to their large beam hole diameter, as well as their smooth shape, their impedance was expected to be lower than that of the copper cavities with smaller openings and re-entrant nose cones. Since the bunch current in LEP is often limited by collective effects, it was important to measure the impedances of these cavities to verify these calculations. Furthermore, the energy lost in the SC cavities can lead to an excessive heat load of the cryogenic system, and it is hence desirable to reduce it as much as possible by the higher-order-mode (HOM) couplers in each cavity. Also two Ferrite absorbers were recently installed tentatively near two SC cavity modules, in order to reduce the energy leaking into neighbouring cavities.

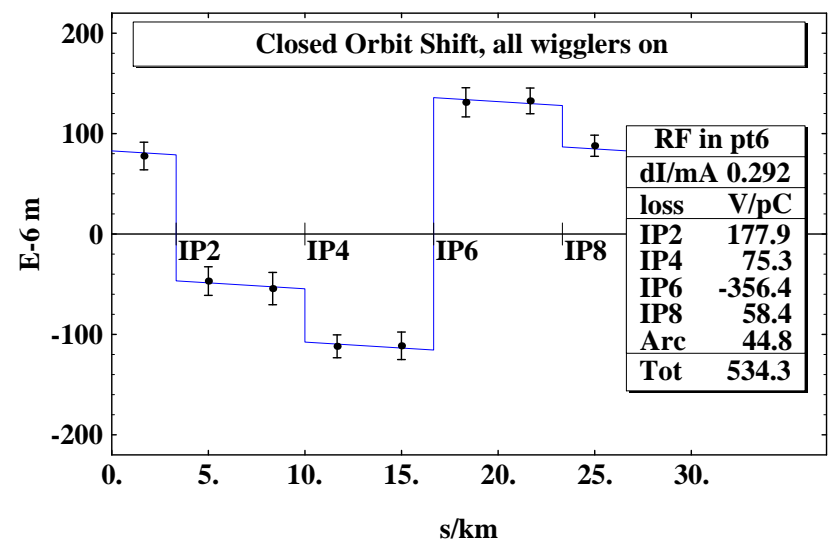

Figure 1: Closed orbit shift as function of position.

\section{THE CALCULATED MODE LOSS PARAMETERS OF THE CAVITIES}

The impedances of the copper and SC cavities, as well as that of inter-cavity and inter-module bellows, have been calculated extensively using a variety of computer programs such as ABCI[5]. The results are usually expressed by the "longitudinal loss factor" $k_{\|}(\sigma)$ which is given by the integral over the product of the impedance and the power spectrum of the bunch current. It is therefore a function of the bunch shape - which is well approximated by a Gaussian in LEP - and of its rms length $\sigma$. For a typical bunch length of $\sigma=10 \mathrm{~mm}$, one SC cavity has a loss factor of $k_{\|}=1.21 \mathrm{~V} / \mathrm{pC}$, while a Cu cavity has $k_{\|}=2.86$ $\mathrm{V} / \mathrm{pC}$. The loss factors are plotted in figure 2 as a function of bunch length. The loss factor of all other elements are much smaller and can be found in the LEP Design Report [6] as well as in a data base which is updated whenever the 


\begin{tabular}{|c||c|c|c|c|}
\hline cavity type & Point 2 & Point 4 & Point 6 & Point 8 \\
\hline \hline $\mathrm{Cu}$ & 60 & - & 60 & - \\
\hline $\mathrm{Nb}$ & 16 & - & - & - \\
\hline $\mathrm{Nb}-\mathrm{Cu}$ & 16 & 56 & 32 & 56 \\
\hline
\end{tabular}

Table 1: Distribution of the cavities in LEP.

number of installed cavities changes [4]. At the time of the experiment (November 1996), a total of $120 \mathrm{Cu}$-cavities, with a beam hole of $50 \mathrm{~mm}$ radius, and $176 \mathrm{SC}$ cavities with a beam hole of $120 \mathrm{~mm}$ radius were installed in LEP. Among the SC cavities, the majority (160) are of the Nb$\mathrm{Cu}$ type, and 16 of the full $\mathrm{Nb}$ type (in Point 2), but their geometries - and hence their loss factors - are essentially the same. All these cavities are located in dispersion-free straight sections, at both sides of the even numbered interaction points. Their distribution is shown in table 2.

\section{THE EXPERIMENT}

\subsection{The beam measurement}

The energy loss of a bunch along the circumference of LEP was determined by measuring the closed orbits for different bunch currents with the beam position monitors (BPMs) at locations of finite dispersion. In the LEP lattice, only the (eight) arcs have nonzero dispersion, and BPMs are installed at each D-quad where for the present optics $D_{x}=0.597 \mathrm{~m}$. The deviation of a bunch from the nominal energy can be obtained from the measured orbit change $\Delta x_{c o}$ through the relation $\Delta x_{c o}=D_{x} \Delta E / E$. This measurement depends critically on the stability of the closed orbit, and one therefore loses accuracy when measuring only after changing the current in a bunch which takes time. Therefore a method has been developed which simultane-

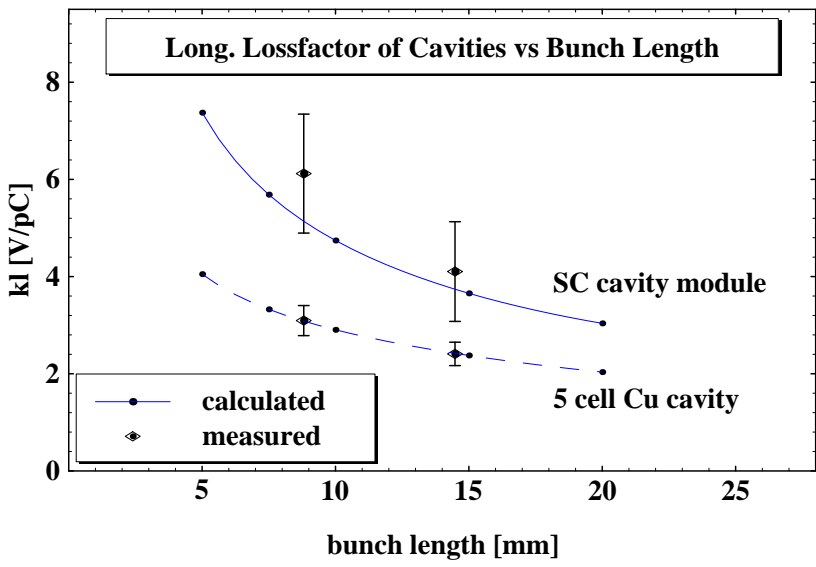

Figure 2: Calculated and measured loss factors versus bunch length for $\mathrm{Cu}$ and $\mathrm{SC}$ cavities in LEP.

ously measures the orbits of up to four bunches with different currents. Then the orbits are subtracted from each other in order to calculate the loss factors. However, the difference orbits show some distortion due to small betatron oscillations, which may be excited by energy losses at finite dispersion. Also the rather strong tune dependence on current could lead to a distortion [7]. To minimize the influence of these effects, the measurements in each arc extending over several betatron wave lengths - were averaged individually.

The bunch lengths were adjusted with two sets of wiggler magnets, which are called "damping wigglers" and "polarization wigglers". The measurements were performed for $\sigma=14.5,8.8$ and $2.7 \mathrm{~mm}$. For the two cases of long bunches with one or both wigglers excited, bunch lengthening by collective effects is expected to be negligible up to a bunch current of $0.45 \mathrm{~mA}$. The shortest bunches are obtained without wigglers, but then the maximum current which could be stored was limited to $0.1 \mathrm{~mA}$. For this case, some bunch lengthening is expected already at that value. Such low currents reduce the accuracy of the measurements - fortunately, they are not important for LEP operation.

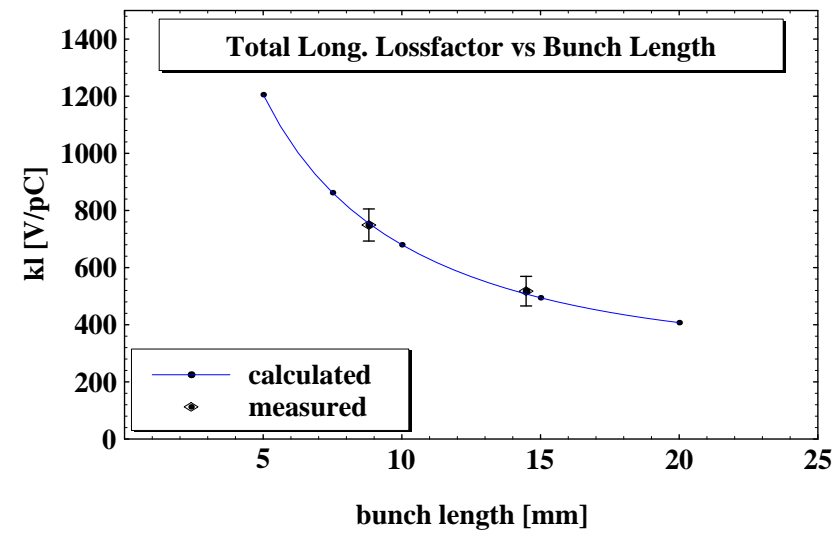

Figure 3: Calculated and measured total loss factor versus bunch length.

The RF cavities were powered only in point 2 or in point 6 , which allows measuring the energy losses in the three other points where RF cavities are installed. The passive SC cavities were detuned in order to avoid a large energy loss due to excitation of the fundamental mode ("beam loading") - which is normally compensated by increased RF power when the cavities are excited. Then the beam induced voltage is sufficiently small to be neglected.

\subsection{Temperature measurements at the cavities}

The energy lost by the beam, which is not eliminated by the HOM couplers or travelling down the beam tube, appears as a heat load in the module[8]. The calorimetric measurements of the heat load correspond to only about $1 \%$ of the total beam losses. The dynamic load dependence of beam induced heating for a module can be described as an impedance, which surprisingly depends on beam energy. The power loss is $0.9 \mathrm{MW}$ at the injection energy of $22 \mathrm{GeV}$ 


\begin{tabular}{|c|c|c|c|c|c|c|}
\hline$\sigma$ & arcs & pt.2 & pt.4 & pt.6 & pt.8 & total \\
\hline $\mathrm{mm}$ & $\mathrm{V} / \mathrm{pC}$ & $\mathrm{V} / \mathrm{pC}$ & $\mathrm{V} / \mathrm{pC}$ & $\mathrm{V} / \mathrm{pC}$ & $\mathrm{V} / \mathrm{pC}$ & $\mathrm{V} / \mathrm{pC}$ \\
\hline \hline 14.5 & 26.2 & 178.9 & 69.5 & 186.8 & 56.1 & $\mathbf{5 1 7 . 6}$ \\
\hline 8.8 & 68.5 & 241.8 & 95.1 & 248.1 & 96.1 & $\mathbf{7 4 9 . 2}$ \\
\hline 2.7 & 113.6 & -485.9 & 54.9 & 257.0 & 60.5 & 742.9 \\
\hline
\end{tabular}

Table 2: Measured longitudinal loss factors for arcs, RFsections and the whole machine for different bunch lengths.

and 4.2 MW at the operating energy of $86 \mathrm{GeV}$. Temperature measurements of the cold inter-cavity bellows and of the warm intermodule bellows have been made. The power loss in the warm, $100 \mathrm{~mm}$ diameter bellows is $0.08 \mathrm{MW}$ at $22 \mathrm{GeV}, 0.17 \mathrm{MW}$ at $45 \mathrm{GeV}$, and $0.35 \mathrm{MW}$ at $86 \mathrm{GeV}$. For the cold bellows with $200 \mathrm{~mm}$ diameter we found $0.13 \mathrm{MW}$ at $46 \mathrm{GeV}$ and $0.18 \mathrm{MW}$ at $86 \mathrm{GeV}$. The bellows temperature along a straight section shows an exponential decrease with distance from the modules.

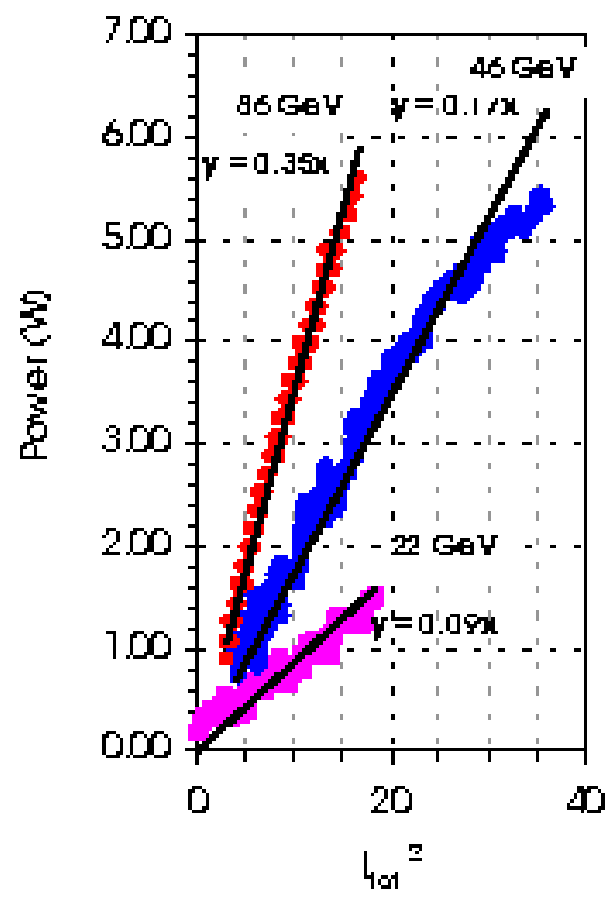

Figure 4: Dissipated power in intermodule bellows.

\section{RESULTS}

The results of the orbit measurements as function of current are shown in figure 1 for the case of $\sigma=14.5 \mathrm{~mm}$ and for powering the cavities in point 6 . The sudden drop of the orbit displacement caused by the loss in the cavities in points 2,4 and 8 , is clearly visible. Since the dispersion is close to zero in the long straight sections where the cavities are located, the orbit change can only be observed in the adjacent arcs. In the 8 arcs, one can see a small drift of the orbit towards the inside, which allows estimating the distributed impedance. This is mainly caused by the shielded bellows, of which there are a large number (2800) in the arcs, and to a lesser degree by the larger unshielded bellows, the holes to the pumping chamber, and by the nonzero wall resistivity. The cavities in point 6 replace the energy lost in the rest of the ring and makes a jump to the outside.

From this and other measurements we get the orbit change per unit bunch current $d x_{c o} / d I_{b}$. Then the longitudinal loss factors of all 4 cavity sections and of the arcs can be obtained from the expression

$$
k_{\|}=\frac{f_{0} E}{e D_{x}} \frac{\Delta x_{c o}}{\Delta I_{b}}
$$

with $f_{0}$ is the revolution frequency, $E$ the beam energy, and $D_{x}$ the horizontal dispersion. The loss factors for the arcs, the four RF-sections and the complete machine for different bunch lengths (obtained by different wiggler settings) are listed in table 2.

These loss factors contain not only the effect of all the cavities in one long straight section but also the one of the bellows located there. The factors of the latter were subtracted to obtain the ones of the $\mathrm{Cu}$ and superconducting cavities alone. These measurement are show in figure 2 together with the calculations. The agreement is very good. Finally the measured total loss factor of LEP is plotted in figure 4 showing also good agreement.

\section{REFERENCES}

[1] D. Brandt, P. Castro,K. Cornelis, A. Hofmann, G. Morpurgo, G.L. Sabbi, J. Wenninger, B. Zotter; Proceedings of the 1995 Particle Accelerator Conference.

[2] D. Brandt, K. Cornelis, V. Danilov, A. Hofmann, J.C. Juillard, E. Perevedentsev, E. Peschardt, E. Rossa, F. Tecker, D. Wang, B. Zotter; Proceedings of the 1993 Particle Accelerator Conference, p 3429

[3] C. Zhang; KEK Preprint 96-88.

[4] O. Brüning;"An Impedance Data Base for LEP and LHC”, contribution to this conference.

[5] Y. Chin: User's Guide for ABCI, CERN SL/94-02 (1994).

[6] LEP Design Report, Vol. III LEP 2, ed. C. Wyss, CERNAC/96-01 (1996).

[7] S. Heifets; private communication.

[8] G. Cavallari, P. Gayet, G. Geschonke, M. Jimenez, S. Myers: CERN LEP2 note 97-40 (1997). 